\title{
The Effects of Continuous Labor Support by Midwife: A Randomized Controlled Trial
}

\author{
Gulsemin Bostanoglu ${ }^{1}$, Meltem Demirgoz Ba ${ }^{2}{ }^{[C}$ \\ ${ }^{1}$ Department of Midwifery, Institute of Health Sciences, Marmara University, Turkey \\ 2 Department of Midwifery, Health Sciences Faculty, Marmara University, Turkey. \\ Correspondence Author: Meltem Demirgoz Bal \\ E-mail: meltemdemirgoz@gmail.com \\ Received: $12.05 .2020 \quad$ Accepted: 29.05 .2021
}

\begin{abstract}
Objective: The aim of this study was to evaluate the effects of continuous labor support by midwife.

Methods: This study was a randomized controlled experimental design. Seventy primipara women were randomized into two groups, the continuous labor support by midwife group $(n=35)$ and control group $(n=35)$. In the experiment group was given the continuous midwife support and in the control group were undergone to routine hospital care in the clinic. Visual Analog Scale was used to assess women's anxiety level and coping with labor pain. All interventions and childbirth time were recorded by the researcher.

Results: Anxiety level decreased and the level of coping with labor pain increased in the experimental group $(p<0.05)$. The total childbirth time in the intervention group was shorter by 2 hours and 21 minutes. Labor induction, episiotomy, amniotomy and perineal trauma were higher in the control group ( $p<0.05)$.

Conclusion: Continuous labor support should be provided at each childbirth to enhance childbirth outcomes.

Keywords: Continuous labor support, randomized controlled study, midwife, childbirth, Turkey
\end{abstract}

\section{INTRODUCTION}

Association for the Improvement in Maternity Services (AIMS) in the United Kingdom, proposed the definition of normal childbirth the presence or absence of intervention. According to this definition, the childbirth is divided into three categories.

1. Operative or interventions childbirth (caesarean section, forceps, vacuum),

2. Obstetric childbirth (artificial rupture of membranes (ARM), prostaglandin gel, labor induction, and episiotomy)

3. Normal childbirth (1).

Normal childbirth should occur spontaneously and without intervention, and it is a process in which the mother and baby continue to live healthy (1). Thus, normal childbirth should not have a serious burden on the national economy.
According to this classification, when examining Turkey's childbirth data, unfortunately the situation does not seem very pleasant. The first group of the operative/interventions childbirth, Turkey with $52 \%$ incidence of caesarean section rate is the country with the highest caesarean section rates in the Organization for Economic Co-operation and Development (OECD). At the same time, attempts to have normal childbirths after caesarean section are limited. Thus, these high rates remain constant within the framework of a "once caesarean section, always caesarean section" philosophy. Also, it is known that elective caesarean section causes very high childbirth costs in countries (2).

Obstetric childbirth rate as a second group in the AIMS classification, is around $45 \%$ in Turkey (2). Childbirths are performed vaginally, episiotomy applications and are often carried out using labor induction. Moreover, in Istanbul as one of Turkey's most developed and most crowded city is given the birth services by obstetricians. Although midwives 
are graduated with the skills and knowledge to manage the antenatal, innatal and postnatal processes during their fouryear bachelor education, midwives in the maternity unit are often interested in documentation and organization. Whereas it is more desirable for obstetricians to participate in the management of childbirth in cases of deviation from normal and to develop themselves in this field. While obstetricians spend their effort and time by playing an active role in the normal childbirth process, but also midwives who are specially trained for this field are losing ability over time in our country. For this reason, it is not possible to give birth support to women without interruption in our country, and this care is provided by different people. Besides communication with the pregnant woman is only during examinations (dilatation, effacement, and so on.) and a very short period of communication is established with the woman. It is beyond doubt that the cost of this situation is very high.

In the last group of normal childbirth rate in Turkey it is very low. Very high-income families manage the process by receiving continuous labor support. Continuous labor support is included physical support, emotional support, information support and advocacy during labor and childbirth (3-5). Physical support is the act of directly assisting the woman in physical terms (using non-pharmacological methods of coping with pain, helping her to stand up, and so on.). Emotional support is the using expressions of emotional support, love and respect, and also contains that making them feel by their side in the whole process, making them express their feelings and establishing sentences that empower, praise and motivate the woman. Information support is providing direct information (such as duration of childbirth, effacement, dilatation, fetal head level) and positive feedback, and advocacy support refers to advocacy for women's rights on behalf of women. Also, continuous labor support includes that allowing her to stand up unless there is a risk and supporting oral intake. Besides, for the support provided to be continuous, it should cover at least $80-85 \%$ of the childbirth process and should be given by the same person (s) as much as possible (6).

This study was planned as a randomized controlled study to investigate the effects of continuous labor support. In this study, it is important because midwives have the potential to determine the effects on the labor process when they are included in the birth.

\section{METHODS}

\subsection{Specific Objective/Hypothesis:}

$\mathrm{H} 1$ : There is a difference in the anxiety level between the experiment group and the control group.

$\mathrm{H} 2$ : There is a difference in the level of coping with labor pain between the experiment group and the control group.
H3: There is a difference between in the interventions childbirth the experiment group and the control group.

\subsection{Design:}

The study was approved by the Istanbul Medeniyet University Ethics Committee of Clinical Research of Goztepe Training and Research Hospital (Date: 26.10.2017, Number:0293). Firstly, after participant's hospitalization, pregnant women were informed about the study by the researcher. Experiment or control group discrimination was provided for those who agreed to participate in the study. The trial was reported according to the CONSORT guidelines (Figure 1).

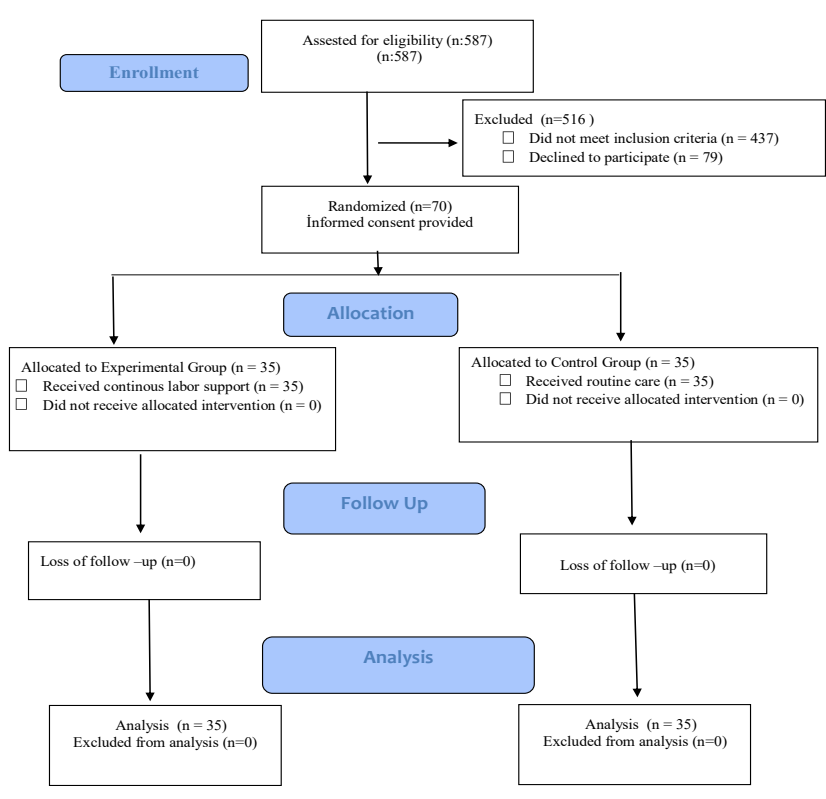

Fig. 1: CONSORT flow diagram

Data were collected from primipara pregnant women who were recruited from the Gynecology and Obstetrics Clinic of Goztepe Training and Research Hospital between November 2017 and May 2018, through a randomized controlled experimental study. The aim of this study is to examine the effect of continuous labor support on childbirth outcomes. With a power of 0.8 and an acceptable Type I $\alpha$ error size of 0.05 , each group required 35 participants. The women were single-blind randomized into two groups, the experiment and control.

The primary aim of this study was to evaluate the effect of continuous labor support anxiety level and coping with labor pain level using Visual Analog Skala (VAS). The validity and reliability of VAS in all cultures has been established and is also a very easily applicable scale (7). VAS is a valid and reliable scale in all cultures and can be applied very easily. In this study, a $10 \mathrm{~cm}$ VAS was used and based on the woman's hand marking. Childbirth interventions and perineal trauma related to childbirth were made by marking "present" or "absent". Childbirth time recorded by researcher. The research flow is given in Figure 2. 
Experiment and Control Group Randomization: (https://randomizer.org/)

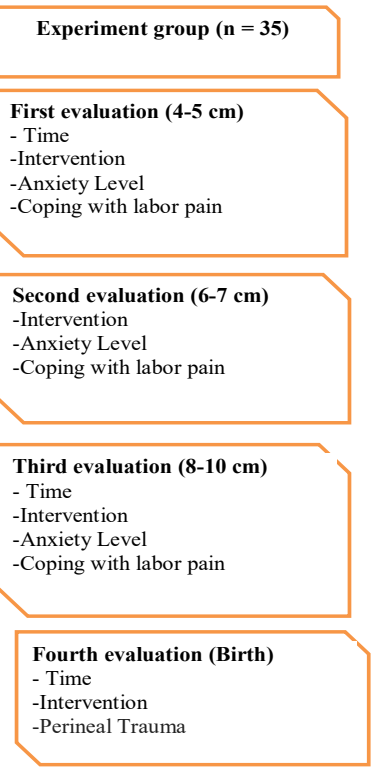

Fig. 2: Research Flow Chart

Care services are provided in reserved rooms for every pregnant woman in this hospital. The women in the experiment group were given individualized continuous labor support consisting of four components from the active phase to the birth moment. Furthermore, it was ensured that the majority of the women's time was spent with the midwife. Emotional support was tried to be given, in order to create a feeling of being safety atmosphere and comfort in the pregnant woman, to make the woman feel that she was being cared for and to create the feeling that she was in control of the process. For physical support the birth room was made darker and the labor created as quiet an environment as possible during the care. Therapeutic touch, hand holding, standing, moving and deep breathing exercises were performed. For information support, information was shared with the woman about the dilatation, effacement, and birth process. The advocacy component contained the protecting the pregnant woman, understanding her needs, taking care of her privacy, and respecting her, helping her make health-care choices (for example, not to let routine connecting to the NST, allowing her to freedom of movement, allowing her to drink water, etc.).

The length of the active phase at birth should not exceed 12 hours in nullipars, and the length of the transition phase should not exceed 3 hours. The second stage of labor should not exceed an hour and the third stage should not exceed half an hour (1). Pregnant women in the control group were also cared for by the same midwife from the active phase from the active phase to the childbirth accordance with hospital protocols. Hospitals in Turkey do not routinely use nonpharmacologic methods to reduce labor pain. According to this hospital protocol in routine care, information was shared after each examination and deep breathing exercises were provided during contractions. Dilatation and effacement are determined at regular intervals for women in the control group. NST and induction are frequently used. Oral intake is not allowed and there is often no freedom to change positions.

\subsection{Inclusion and Exclusion Criteria:}

All primipara pregnant women included in this study did not attend antenatal education classes. However, as inclusion criteria;

- Started labor process and hospitalized,

- Term and singleton pregnancy,

- A live/healthy fetus,

- Spontaneous vaginal delivery,

- Longitudinal and vertex position of the fetus,

- $\quad$ Active phase (dilatation $=4 \mathrm{~cm}$ and above),

- Body Mass Index (BMI) under 30 and are estimated,

- Ultrasonographic baby weight $<4 \mathrm{~kg}$,

- Who had not previously been diagnosed and treated for anxiety,

- Who agreed to participate,

- Given consent were included in the study.

Exclusion Criteria;

- Maternal and fetal complications,

- Pregnant women who have a head-pelvic noncompliance,

- A fetal hypoxia (distress),

- Early Membrane Rupture (EMR),

- Risk of fetal anomaly,

- Have any language-communication problems in pregnant women,

- Discontinuation of the study at any stage of the study,

- Cesarean section

\subsection{Randomization:}

The random.org site was used to determine which women were included in the control group or in the experiment group. The number of experiment and control groups determined by random.org is as follows.

Numbers experiment: 22, 19, 51, 26, 2, 63, 39, 61, 32, 11, 56, $64,35,37,25,66,6,8,31,29,34,42,59,48,16,58,24,67$, $70,18,38,53,45,13,28$ th pregnant women.

Number control: 23, 4, 49, 54, 46, 43, 36, 62, 41, 21, 65, 30, $5,14,68,50,7,10,20,44,60,12,57,3,55,47,27,15,17,69$, $9,71,1,40,33,52$ nd in the pregnant women. 


\subsection{Blinding (masking):}

First of all, pregnant women who meet the inclusion criteria of the study were directed to the researcher by a physician who did not know the aim of the study. Afterwards, necessary explanations were made to each woman by the researcher. Written and verbal consents of the women who accepted to participate in the study were obtained at this stage. After approval, the women were included in the experiment or control group according to the previously determined and above-mentioned numbers. Experiment and control groups were not given maintenance simultaneously. After each woman's childbirth, the other pregnant women were included in the study. The data obtained were entered into SPSS trial program by researcher. The analyses were performed by a statistician without specifying the experiment and control groups in order to prevent possible bias.

\subsection{Statistical Analysis:}

The demographic and clinical characteristics of the experiment and control groups were compared with the chisquare test. Anxiety level, coping with labor pain levels and childbirth time were evaluated with Mann-Whitney $U$ Test and interventions and perineal trauma were evaluated with chi-square test.

\subsection{Measurement and Instruments:}

The data collection flow is detailed in Figure 2. Childbirths are this hospital are performed by obstetricians. In this study, obstetricians were not informed about the study to prevent possible biases. Anxiety level and coping with labor pain were determined with VAS. Childbirth time and interventions were recorded at intervals. The fee to be paid when the participants were discharged was obtained from the patient records.

\section{RESULTS}

Demographic characteristics of the women participating in the study are given in Table 1.

Table 1. The characteristics of the participants $(n=70)$

\begin{tabular}{|c|c|c|c|c|c|c|c|}
\hline \multirow{2}{*}{\multicolumn{2}{|c|}{ Demographic Characteristics }} & \multirow{2}{*}{\multicolumn{2}{|c|}{ Experiment Group ( $n=35$ ) }} & \multirow{2}{*}{\multicolumn{2}{|c|}{ Control Group (n=35) }} & \multicolumn{2}{|c|}{ Analysis } \\
\hline & & & & & & & \\
\hline \multicolumn{2}{|l|}{ Age } & $24,11 \pm 3,91$ & 24,00 & $23,86 \pm 4,31$ & 23,00 & 21,945 & 0,145 \\
\hline \multicolumn{2}{|l|}{$\begin{array}{l}\text { Marriage Period } \\
\text { (months) }\end{array}$} & $19,86 \pm 9,25$ & 18,00 & $20,51 \pm 9,92$ & 18,00 & 7,546 & 0,580 \\
\hline \multicolumn{2}{|l|}{ BMI } & $28,19 \pm 3,18$ & 28,80 & $29,12 \pm 3,96$ & 28,6 & 50,000 & 0,394 \\
\hline \multirow{2}{*}{ Family Type } & Nuclear Family & 30 & 85,7 & 32 & 91,4 & \multirow{2}{*}{0,439} & \multirow{2}{*}{0,355} \\
\hline & Extended Family & 5 & 14,3 & 3 & 8,6 & & \\
\hline \multirow{2}{*}{ Educational level } & Under 8 year & 15 & 42,8 & 20 & 57,1 & \multirow{2}{*}{0,557} & \multirow{2}{*}{0,557} \\
\hline & Over 9 years & 20 & 57,2 & 15 & 42,9 & & \\
\hline \multirow{3}{*}{ Economic level } & Low income & 8 & 22,9 & 5 & 14,3 & \multirow{3}{*}{0,332} & \multirow{3}{*}{0,332} \\
\hline & Average Income & 8 & 22,9 & 5 & 14,3 & & \\
\hline & High income & 19 & 54,3 & 25 & 71,4 & & \\
\hline
\end{tabular}

There was a statistically significant difference between the experiment and control groups anxiety level and coping with labor pain ( $<0.01$ ) (Table 2 ).

Table 2: Comparison of anxiety level and coping with labor pain of the study groups $(n=70)$

\begin{tabular}{|l|c|c|c|c|c|c|}
\hline \multirow{2}{*}{ Anxiety Level } & \multicolumn{5}{|c|}{ Group } & \multicolumn{2}{c|}{} \\
\cline { 2 - 7 } & \multicolumn{3}{|c|}{ Experiment } & \multicolumn{2}{c|}{ Control } & \multicolumn{2}{c|}{ Analysis } \\
\cline { 3 - 8 } & $\mathbf{Z}_{\text {Mwu }}$ & $\mathbf{p}$ \\
\hline Dilatation (4-5 cm) & 5,63 & 1,65 & 4,77 & 1,65 & $-2,090$ & 0,307 \\
\hline Dilatation (6-7 cm) & 3,83 & 0,82 & 6,71 & 1,51 & $-6,453$ & $<0,001$ \\
\hline Dilatation (8-10 cm) & 2,00 &, 84 & 9,12 & 1,23 & $-7,261$ & $<0,001$ \\
\hline Coping with Labor Pain & & & & & & \\
\hline Dilatation (4-5 cm) & 4,82 & 2,20 & 5,14 & 2,30 &,- 560 & 0,575 \\
\hline Dilatation (6-7 cm) & 6,60 & 1,56 & 4,06 & 1,49 & $-5,620$ & $<0,001$ \\
\hline Dilatation (8-10 cm) & 8,69 &, 83 & 2,44 & 1,74 & $-6,862$ & $<0,001$ \\
\hline
\end{tabular}

Mann-Whitney U Test; Data are represented as mean \pm standard deviation
There was a statistically significant difference between the experiment and control groups such as amniotomy and labor induction. None of the participants in the experiment group developed perineal trauma related to childbirth. A total of 15 women in the control group (42.9\%) were determined perineal trauma $(p<0.01)$ (Table 3$)$.

Table 3. Comparison of participant labor interventions $(n=70)$

\begin{tabular}{|c|c|c|c|c|c|c|c|}
\hline \multirow{2}{*}{\multicolumn{2}{|c|}{ Labor Interventions }} & \multicolumn{4}{|c|}{ Intervention } & \multirow{2}{*}{\multicolumn{2}{|c|}{ Analysis }} \\
\hline & & \multicolumn{2}{|c|}{ Yes } & \multicolumn{2}{|c|}{ No } & & \\
\hline \multirow[t]{2}{*}{ Labor induction } & Experiment & - & - & 35 & 100 & \multirow{2}{*}{66,111} & \multirow{2}{*}{$<0,001$} \\
\hline & Control & 34 & 97,1 & 1 & 2,9 & & \\
\hline \multirow{2}{*}{ Episiotomy } & Experiment & 27 & 77,1 & 8 & 22,9 & \multirow{2}{*}{2,696} & \multirow{2}{*}{$0,094^{a}$} \\
\hline & Control & 32 & 91,4 & 3 & 8,6 & & \\
\hline \multirow{2}{*}{ Amniotomy } & Experiment & 2 & 5,7 & 33 & 94,3 & \multirow{2}{*}{38,445} & \multirow{2}{*}{$<0,001^{\mathrm{a}}$} \\
\hline & Control & 27 & 74,9 & 8 & 25,1 & & \\
\hline \multirow[t]{2}{*}{ Perineal Trauma } & Experiment & - & - & 35 & 100 & \multirow{2}{*}{19,091} & \multirow{2}{*}{$<0,001$} \\
\hline & Control & 15 & 42,9 & 20 & 57,1 & & \\
\hline
\end{tabular}

${ }^{a}$ Chi-square test 
The total childbirth time in the intervention group was shorter by 2 hours and 21 minutes $(p<0.01)$ (Table 4$)$.

Table 4. Comparison of participants in terms of childbirth time $(n=70)$

\begin{tabular}{|l|l|l|l|l|l|l|}
\hline \multirow{2}{*}{ Childbirth Time } & \multicolumn{4}{|c|}{ Group } & \multicolumn{2}{c|}{ Analysis } \\
\cline { 2 - 7 } & Experiment & \multicolumn{2}{c|}{ Control } & \multicolumn{2}{c|}{} \\
\cline { 2 - 7 } & Mean & SD & Mean & SD & $Z_{\text {MwU }}$ & $p$ \\
\hline Time from $4 \mathrm{~cm}$ to $8 \mathrm{~cm}^{\mathrm{b}}$ & $1: 55$ & $0: 35$ & $3: 17$ & $1: 34$ & $-4,008$ & $<0,001^{\mathrm{a}}$ \\
\hline Time from $8 \mathrm{~cm}$ to $10^{\mathrm{b}}$ & $1: 08$ & $0: 23$ & $1: 47$ & $0: 47$ & $-3,950$ & $<0,001^{\mathrm{a}}$ \\
\hline Childbirth & $0: 23$ & $0: 07$ & $0: 47$ & $0: 28$ & $-3,955$ & $<0,001^{\mathrm{a}}$ \\
\hline Plasental delivery & 0,39 & $0: 15$ & $0: 35$ & $0: 12$ & $-3,486$ & $<0,001^{\mathrm{a}}$ \\
\hline Total childbirth time $^{\mathrm{b}}$ & $3: 25$ & $0: 55$ & $5: 46$ & $2: 27$ & $-4,656$ & $<0,001^{\mathrm{a}}$ \\
\hline
\end{tabular}

aMann-Whitney U Test; ${ }^{b h h: ~} \mathrm{~mm}$ (hour: minute)

The only limitation of this study is that it is performed with primipara women. Furthermore, the study results may change when multiparous women are included.

\section{DISCUSSION}

According to Tumbilin and Simpkin (8), psycho-biological reactions such as anxiety and fear in pregnant women come into play in cases of fear, embarrassment, frustration, restriction, and feeling disrespected and ignored at childbirth process. These reactions may be reversed during the intrapartum period by giving the woman continuous support. As a matter of fact, in our study, anxiety significantly reduced in women receiving continuous labor support. Similar to our study, Bohren et al. (6), Dickinson et al. (9), Hodnett (10),Sangestani et al. (11), Sehhatie et al. (12), Sydsjö et al. (13), and determined that the continuous one-to-one support reduces anxiety in childbirth. In addition, in a study reported that birth support is the most effective factor in reducing anxiety at childbirth after epidural anesthesia (14). Moreover, in a meta-analysis study was reported that the positive effects of labor support at childbirth on anxiety and depression continued in the postpartum period (15).

When anxiety is not controlled during intrapartum period, it causes epinephrine release (16). Increased epinephrine induces peripheral and uterine vasoconstriction and so causing anoxia in the tissues and increasing the pain sensation. Therefore, continuous labor support provided to the woman in labor process may be effective in coping with labor pain by controlling anxiety. Labor pain is subjective and affected by a wide variety of factors. Therefore, it is recommended that to evaluate the levels of coping with labor pain rather than pain levels $(10,16)$. As a matter of fact, in this study, the mean scores of coping with labor pain in the experiment group receiving continuous labor support increased statistically. This result is in parallel with the findings of many studies in the literature $(16,17)$. Support given at this stage of childbirth, it makes a significant difference in the level of coping with labor pain in women.

In this study, the effect of labor support on interventions during childbirth process was evaluated in terms of using of labor induction (oxytocin), forceps, vacuum, episiotomy, epidural analgesia and artificial rupture of membranes. There was a statistically significant difference between the experiment and control groups in using of labor induction and artificial rupture of membranes $(p<0.01)$. In the metaanalysis of randomized controlled studies by Hodnett et al. (10) was found that continuous labor support reduced interventions in labor process, similar to our study. However, Sandall et al. (18) and Man Wang et al. (19), unlike these results, they did not detect any change in the intervention rates in the group they gave birth support.

In Turkey, the use of labor induction is almost routine, and it is an extremely costly workload that directly increases the cost of normal childbirth. It is a remarkable fact that the continuous labor support determined in this study reduces the need for oxytocin. In fact, although labor induction was applied to all women (97\%) in the control group, total childbirth time was longer than the intervention group. These results is compatible with the results of Sehhatie's study results (12). Continuous and appropriate midwifery care plays the role of oxytocin. Demirel and Celik (20) stated that the nature childbirth was intervened by drawing attention to the complications of the labor induction. It is reported that the only cost of labor induction is met in cases when labor is completed vaginally, and when the Bishop Score has 6 points and above, however except this situation induction is only cost increasing (21). For this reason, it has been suggested to take into consideration patient/physician preferences as well as the cost of induction of labor (22). However, in another recent study by Alfirevic et al. stated that the using of the low-dose oral misoprostol instead of oxytocin has been reported to be more cost-effective in induction of labor (23). In a study that supported this view, was found that the using of the misoprostol was found to be much more costeffective than using oxytocin-containing fluids (24). For this reason, in addition to patient/physician preferences, current and evidence-based cost-effective interventions should not be ignored.

Episiotomy is a routine intervention performed today, to facilitate the childbirth of the fetus especially in the first pregnancy and to prevent postpartum perineal trauma in women $(25,26)$. Sandall et al. $(18)$ in the meta-analysis similar to our study, found that continuous birth support provided a reduction in the rate of episiotomy. As determined in our study, routine episiotomy, especially in the primipara pregnant women is performed in our country. Therefore, continuous birth support did not change the rates of routine episiotomy. Failure to achieve this change can be explained not by the ineffectiveness of continuous midwifery support provided, but also by the fact that using of the routine habits and gynecologists are more likely to be more effective in the management of the childbirth process.

In this study, none of the participants in the experiment group occurred perineal trauma. Insisting on the limitation of the duration of the second stage of labor, also obliges the person assisting the delivery to intervene the process and promptly obliges to try to terminate the delivery immediately 
if the desired time limit is exceeded. Limiting the duration of the second stage in birth process increases the $\mathrm{pH}$ of the newborn, but this may lead to more interventions in childbirth. Therefore, every intervention causes perineal area integrity to deteriorate $(25,26)$. The results of the metaanalyses in the current literature, similar to our results, were determined that continuous labor support was effective in maintaining the integrity of the perineal area (18). In addition, various degrees of perineal damage (due to prevention of vertical positions, limitation of mobility, the induction of labour, and so on.) repair of these perineal injuries and the use of antibiotics increased the direct cost of childbirth in the control group. In the short term, increasing the workload of health personnel directly, delaying the discharge of the woman from the hospital, interrupting the mother-infant connection and increasing future breastfeeding problems may affect the cost of birth. In the long term, perineal trauma associated with labor may lead to chronic incontinence and dyspareunia, which may adversely affect the quality of life and self-confidence, leading to additional costs (27). However, a study by Sundquist (28) determined that $45 \%$ of perineal traumas caused additional various complaints (incontinence, hemorrhage, infection, and so on.), leading to additional costs to the system over a period of 4-8 years. Therefore, in the cost calculations of perineal traumas, the pricing approach only for the period from the hospital to discharge may lead to the ignorance of the magnitude of the cost $(29,30)$. In addition, from social aspects, social stigmatization, prolonged separation from the spouse and also the presence of negative emotional feelings may lead to health seeking behaviors and therefore that this situation returns to the health system as a cost (31). WHO reports that perineal trauma related to childbirth affects millions of women whose number is not clearly known each year (32). For this reason, WHO is evaluating episiotomy and obstetric anal sphincter injuries or perineal injuries as health system and health care quality indicators (32-34). World Health Organization has published a report on the prevention of perineal trauma techniques at childbirth is stated that this undesirable outcome can be prevented by giving perineal massage training to in-service trainings to health personnel and this massage style is a low cost intervention. Moreover, it has been reported that perineal trauma would be prevented by this procedure and therefore would be less costly than normal care due to the decrease in the use of suture material and local anesthetic (32).

Although Friedman's labor curve continues to be discussed, it is reported that prolongation of childbirth increases cesarean section rates and interventions at childbirth $(35,36)$. In this research, the total childbirth time in the intervention group shortened by 2 hours and 21 minutes and this situation was parallel to previous studies on continuous birth support (6, 10). As a matter of fact, the shortening of childbirth time in the intervention group reduced the number of interventions and direct costs (EFM, labor induction, enema, amniotomy etc). In addition, the shortening of the childbirth time accelerates the return of the mothers and their babies who cannot be calculated financially to their comfortable homelife, reduces the workload of the midwife/nurse, reduces the risk of exposure to hospital infections and provides more effective use of patient beds (32).

As a result, with continues midwife support, womens' levels of anxiety decreased and levels of coping with pain increased. There has also been a significant reduction in birth interventions. Birth is an unrepeatable and unique experience. For this reason, we can ensure that every woman has a positive birth experience by providing midwife support.

\section{REFERENCES}

[1] Beech BA, Phipps B. Normal birth: women's stories. Downe S, editor. Normal childbirth: Evidence and debate. Edinburgh: Churchill Livingstone; 2004. p.67-79.

[2] TDHS "Turkey Demographic and Health Survey". Hacettepe University Institute of Population Studies. Hacettepe University Institute of Population Studies, T.C. Ministry of Development and TUBITAK. Ankara. Apple Technical Printing; 2018. p.5-8.

[3] NICE. Intrapartum care for healthy women and babies. Clinical Guideline. 2017. (serial online) 3 December 2014 Available from: https://www.nice.org.uk/guidance/cg190.

[4] Hodnett E, Lowe NK, Hannah ME, Willan AR. Effectiveness of nurses as providers of birth labor support in North American hospitals a randomized controlled trial. JAMA 2002; 288(11):1373-1381.

[5] Hodnett ED, Gates S, Hofmeyr GJ, Sakala C. Continuous support for women during childbirth. Cochrane Database Syst Rev 2017; 7(7):CD003766.

[6] Bohren MA, Hofmeyr GJ, Sakala C, Fukuzawa RK, Cuthbert A. Continuous support for women during childbirth. Cochrane Database Syst Rev 2017;6(7):CD003766.

[7] Zemla AJ, Sauer KN, Jarmoszewich K., Wera K, Batkiewitz $\mathrm{S}$, Pietrzykowska M. Measures of preoperative anxiety. Anaesthesiol Intensive Ther 2017; 51(1):64-69.

[8] Tumbilin A, Simkin P. Pregnat women's perceptions of their nurse's role during labor and delivery. Birth 2001;28(1):52-6.

[9] Dickinson JE, Paech MJ, McDonald SJ, Evans SF. Maternal satisfaction with childbirth and intrapartum analgesia in nulliparous labour. Aust N Z J Obstet Gynaecol 2003; 43(6):46310.

[10] Hodnett ED, Gates S, Hofmeyr GJ, Sakala C. Continuous support for women during childbirth. Cochrane Database Syst Rev 2012; 17(10):CD003766.

[11] Sangestani G, Khatiban M, Pourolajal J, Oshvandi K. Influence of doula on the primiparous parturients' anxiety in the delivery ward. J Hayat 2014; 19(4):48-60.

[12] Sehhatie F, Najjarzadeh M, Zamanzadeh V, Seyyedrasooli A. The effect of midwifery continuing care on childbirth outcomes. Iran J Nurs Midwifery Res 2014; 19(3):233-7.

[13] Sydsjö G, Blomberg M, Palmquist S, Angerbjörn L, Bladh M, Josefsson A. Effects of continuous midwifery labour support for women with severe fear of childbirth. BMC Pregnancy Childbirth 2015; 15(1): 2-5.

[14] Fernández-Campos FJ, Escrivá D, Palanca JM, Ridocci F, Barrios C, Gallego J. Women's acute anxiety variations before and after epidural anesthesia for childbirth. J Psychosom Obstet Gynaecol 2017; 38 (2):152-158. 
[15] Scott KD, Berkowitz G, Klaus M. Comparison of intermittent and continuous support during labor: A meta-analysis. Am J Obstet Gynecol 1999; 180(5):1054-9.

[16] Buckley SJ. Physiologic onset of labor and scheduled birth. Buckley SJ, editors. Hormonal physiology of childbearing: Evidence and implications for women, babies, and maternity care. Washington: Connecticut Avenue; 2015. p.14-26.

[17] Safarzadeh A, Beigi M, Salehian T, Khojasteh F, Burayri TT. Effect of doula support on labour pain and outcomes in primiparous women in Zahedan, Southeastern Iran: A randomized controlled trial. J Pain Relief 2012; 1:111-112.

[18] Sandall J, Soltani H, Gates S, Shennan A, Devane D. Midwife-led continuity models versus other models of care for childbearing women. Cochrane Database Syst Rev 2016; 4:CD004667.

[19] Wang M, Song Q, Xu J, Hu Z, Gong Y, Arier C. Lee, Qi Chen. Continuous support during labour in childbirth: a CrossSectional study in a university teaching hospital in Shanghai, China. BMC Pregnancy Childbirth 2018; 18(1):480.

[20] Demirel G, Celik DB. Intervention to natural birth: Use of synthetic oxytocin. Sted 2013; 22(4): 157-164.

[21] Kenny TH, Nicodemo JM, Fenton BW, von Gruenigen, VE. Does quality "bundling" improve obstetrical outcomes? A comparative study of elective inductions. J Reprod Med 2013; 58(9-10):402-410.

[22] Hersh AR, Skeith AE, Sargent JA, Caughey AB. Induction of labor at 39 weeks of gestation versus expectant management for low-risk nulliparous women: a cost-effectiveness analysis. Am J Obstet Gynecol 2019; 220(6):590.e1-590.e10.

[23] Alfirevic Z, Keeney E, Dowswell T, Welton NJ, Medley N, Dias S. Which method is best for the induction of labour? A systematic review, network meta-analysis and cost-effectiveness analysis. Health Technol Assess 2016; 20(65):1-584.

[24] Acharya T, Devkota R, Bhattarai B, Acharya, R. Outcome of misoprostol and oxytocin in induction of labour. SAGE Open Medicine 2017;5: 1-7.

[25] Ergin A. Coping With Labor Pain. Bal MD, Yilmaz SD editors.
Comprehensive birth for midwives. Ankara: Akademisyen Bookstore; 2017. p. 73-75.

[26] Beji NK. Labor. Beji NK editor. Women's health and diseases. Istanbul: Nobel Medical Bookstores; 2015. p. 88-95.

[27] Sinclair AJ, Ramsay IN. The psychosocial impact of urinary incontinence in women. The Obst and Gyna 2011; 13(3):143148.

[28] Sundquist JC. Long-term outcome after obstetric injury: a retrospective study. Acta Obstet Gynecol Scand 2012; 91(6):715-718.

[29] Fitzpatrick M, O'Herlihy C. Short-term and long-term effects of obstetric anal sphincter injury and their management. Curr Opin Obstet Gynecol 2005; 17(6):605-610.

[30] Weledji EP, Elong A, Verla V. Secondary repair of severe chronic fourth-degree perineal tear due to obstetric trauma. J Surg Case Rep 2014; 5:1-3.

[31] Mota RL. Female urinary incontinence and sexuality. Int Braz J Urol 2017; 43(1):20-28.

[32] WHO. Evidence and recommendations. WHO recommendations: Intrapartum care for a positive childbirth experience Geneva: World Health Organization. 2018. p.1835.

[33] Aguiar M, Farley A, Hope L, Amin A, Shah P, Manaseki-Holland $S$. Birt-related perineal trauma in low- and middle-income countries: A systematic review and meta-analysis. Matern Child Health J 2019; 23(8):1048-1070.

[34] Orlovic M, Carter AW, Marti J, Mossialos E. Estimating the incidence and the economic burden of third and fourth-degree obstetric tears in the English NHS: an observational study using propensity score matching. BMJ Open 2017; 12:7(6):1-9.

[35] Bernitz S, Oian P, Rolland R, Sandvik L, Blix E. Oxytocin and dystocia as risk factors for adverse birth outcomes: A cohort of low-risk nulliparous women. Midwifery 2014; 30(3):364-370.

[36] Bugg GJ, Stanley E, Baker PN, Taggart MJ, Johnston TA. Outcomes of labors augmented with oxytocin. Eur J Obstet Gynecol Reprod Biol 2006; 124(1):37-41.

How to cite this article: Bostanoglu G, Demirgoz Bal M. The Effects of Continuous Labor Support by Midwife: A Randomized Control Trial. Clin Exp Health Sci 2021; 11: 251-257. DOI: 10.33808/clinexphealthsci.736497 